Anuario Latinoamericano Ciencias Políticas

y Relaciones Internacionales

vol. 5, 2017

pp. 191-206

\section{¿Ciencia política con sentido social? Colombia, el conflicto armado y los expertos}

DOI: 10.17951/al.2017.5.191

\section{Social sense of political science? Colombia, armed conflict and experts}

\author{
Juan Carlos Rico Noguera * \\ UNIVERSIDAD PONTIFICIA BOLIVARIANA, COLOMBIA \\ $\bowtie$ juan.ricon@upb.edu.co \\ Julián Andrés Caicedo Ortiz ${ }^{* *}$ \\ UNIVERSIDAD DEL CAUCA, COLOMBIA \\ $\triangle$ politologocaicedo@gmail.com \\ Carlos E. Guzmán Mendoza ${ }^{\star * *}$ \\ UNIVERSIDAD DEL NORTE, COLOMBIA \\ $\triangle$ cguzmane@uninorte.edu.co
}

\section{RESUMEN}

Este artículo sostiene que la ciencia política es una disciplina que en medio de su "institucionalización" ha venido tomando un papel cada vez más activo en el proceso de modernización del Estado colombiano a través de su participación en las comisiones de estudio de la violencia, teniendo así un particular "sentido social". Esa certeza hace que el artículo reivindique los estudios disciplinares como un ejercicio que va más allá de narrar de manera épica las trayectorias de una disciplina. En esa medida se aboga por unos estudios disciplinares críticos y rigurosos que puedan dar cuenta de la relación entre experticia e instauración de un proyecto civilizacional como la modernidad. Al final se presentan una serie de cuestionamientos y apuestas metodológicas para superarlos. Cuestionamientos y estrategias se articulan con la apuesta del Grupo de Investigación en Historia de la Ciencia Política en América Latina.

* Profesional en política y relaciones internacionales con maestría en estudios culturales. Profesor catedrático de la Universidad de los Andes, Colombia.

** Politólogo, Universidad del Cauca. Maestro y doctor en estudios latinoamericanos, Universidad Nacional Autónoma de México-UNAM. Candidato a doctor en ciencias sociales, Universidad Autónoma Metropolitana - Xochimilco - UAM-X. Profesor Asociado, Departamento de Ciencia Política Universidad del Cauca, Colombia. Director del Grupo de Investigación GIAPRIP.

*** Doctor en ciencia política, Universidad de Salamanca, España. Profesor Asociado del Departamento de Ciencia Política de la Universidad del Norte, Barranquilla, Colombia. Miembro del Grupo de Investigación en Derecho y Ciencia Política - GIDECP - de la misma Universidad. 
Dossier América Latina: desarrollo y balance de la ciencia política

PALABRAS CLAVE: ciencia política, expertos, conflicto armado, historia de la ciencia, sociología de la ciencia.

\begin{abstract}
This article argues that political science is a discipline that in the midst of its "institutionalization" has started playing an increasingly active role in the process of modernization of the Colombian State through its participation in the commissions for study of violence, thus acquiring a "social sense" in this context. This certainty makes the article authors claim that disciplinary studies are an exercise that goes beyond epic narrating of the trajectories of a discipline. For that reason, critical and rigorous disciplinary studies are required to perceive the relationship between expertise and the establishment of a civilizational project such as modernity. In the end, a series of questions and methodological challenges to be overcome are presented. Questions and strategies are articulated with the commitment and proposal of the Research Group on History of Political Science.
\end{abstract}

KEYWORDS: political science, experts, armed conflict, science history, science sociology.

\title{
Introducción
}

La ciencia política colombiana ha reportado un proceso de crecimiento muy acelerado desde principios de la década de los noventa, un crecimiento que se ha hecho especialmente evidente en el incremento de número de estudiantes de la disciplina, de programas de pregrado y posgrado a nivel nacional, y el incremento de publicaciones científicas (Bejarano, Wills 2005). A lo anterior se suma también una importante cantidad de reflexiones disciplinares que ha permitido entender de alguna manera la historia nacional de la ciencia política, preocupándose especialmente por asuntos como las líneas de investigación, la diferencia entre la ciencia política y los estudios políticos, o la calidad educativa y profesional entre otras cosas (Leyva Botero, Fortou, Preciado, Ramírez 2013). El presente artículo, como parte de los esfuerzos encaminados a la reflexión disciplinar, abordará uno de los aspectos académicos y políticos más importantes de la ciencia política colombiana: su relación con el conflicto armado y, en consecuencia, con la construcción del Estado-nación. ${ }^{1}$

En este artículo se sostendrá que junto con el proceso de modernización ${ }^{2}$, que inició con fuerza el Estado colombiano tras la inauguración del Frente

1 En el caso de que no se entienda cómo es que se relaciona el conflicto armado con la construcción del Estado, se ruega paciencia. Aseguramos que esto se irá aclarando a lo largo del artículo.

2 Entenderemos por modernización el fenómeno mediante el que el Estado adopta las instituciones propias del proyecto liberal-democrático. Esto implica tanto la creación de instituciones 
Nacional ${ }^{3}$, la ciencia política ha venido "institucionalizándose" hasta el punto de ser una fuente importante de expertos/arquitectos del Estado-nación. Lo anterior significa que los politólogos han hecho más que dedicarse a la labor convencional del científico social, pasando del ejercicio exclusivamente académico/universitario al de la concreción de un proyecto histórico y políticamente relevante como lo puede ser el Estado-nación colombiano. En consecuencia, tenemos una disciplina con aparente sentido social. Ahora bien, vale la pena cercar la connotación de la tesis, un tanto grandilocuente, que acaba de enunciarse. Como se verá en unos momentos, aunque el experto tiene por lo general el lugar de un arquitecto cuando el Estado lo requiere, eso no significa que su obra maestra se convierta en un hecho. Por lo general, el experto que se mostrará en este artículo, un experto en la violencia, la democracia y la construcción del Estado, tiene la importante tarea de escarbar el pasado, diagnosticar el presente e imaginar el futuro, pero es difícil establecer hasta qué punto su proyecto ha tenido éxito, o si lo tendrá en algún momento. En ese sentido, lo que puede afirmarse sin el más mínimo temor a la duda, es que los politólogos "expertos en violencia" han venido tomando cada vez más relevancia para el Estado y su proceso de modernización.

Lo anterior puede resultar maravilloso para el politólogo que se encuentre leyendo este artículo, pero no lo es tanto en la medida de que no incluye al conjunto de los politólogos colombianos. Como se verá, una de las virtudes de estudiar la ciencia en sí misma es encontrar que el ejercicio científico, incluido el politológico, oculta relaciones de poder que en este caso concreto hacen que la construcción experta del Estado-nación se haga desde el centro, omitiendo así una multitud de voces y proyectos periféricos.

Reflexiones alrededor de este tipo de realidades son algo que se ha venido intentando con el trabajo del Grupo de Investigación en Historia de la Ciencia Política en América Latina, pero todavía hace falta trabajo y recursos para profundizar en esta materia. Aunque en el caso colombiano hay signos alentadores alrededor de la reflexión disciplinar, como la masiva participación de estudiantes y académicos en los foros y ponencias relacionadas con los estudios disciplinares en el último congreso de ACCPOL (Asociación Colombiana de Ciencia Política) ${ }^{4}$,

capitalistas como la profundización de la participación política. En el plano del funcionamiento del Estado significa una tecnificación del ejercicio de gobierno, algo que lo acerca a la financiación de la ciencia, entre las que se destaca una ciencia para gobierno. Pero específicamente para el caso colombiano significa la imposición del Estado de derecho en todo el territorio nacional, una agenda frustrada hasta el día de hoy.

3 Denominación del periodo que entre 1958 y 1974 caracterizó al sistema político colombiano. Ha sido considerado como uno de los momentos políticos más importantes de la historia política colombiana en la medida en que "pacificó al país" eliminando la violencia bipartidista (Bushnell 2002).

4 Algo no esperado por la organización del evento. En la mesa "Nuevas perspectivas en los estudios sobre el desarrollo de la ciencia política", por ejemplo, el número de participantes excedió significativamente lo previsto. Lo cual es evidencia de la importancia e interés que el tema despierta entre los politólogos y estudiantes de ciencia política.
¿Ciencia política con sentido social? Colombia, el conflicto armado y los expertos

Juan Carlos Rico Noguera Julián Andrés Caicedo Ortiz Carlos E. Guzmán Mendoza 


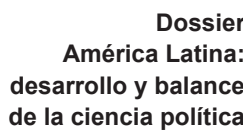

de la ciencia política es todavía difícil recoger los recursos necesarios para realizar una investigación de este tipo.

En aras de la sensibilización sobre la importancia de asumir el estudio de la ciencia como algo relevante para la ciencia política, este artículo se dividirá en cuatro secciones. La primera sección será una muy breve mención de lo que han sido los estudios de la ciencia en general y lo que ha sido en América Latina el estudio de la ciencia política en particular. La segunda sección abordará el estudio de la violencia en Colombia y la implicación progresiva de la ciencia política en ese esfuerzo, mencionando también el lado poco amable de esa implicación. La tercera sección abordará las preguntas que quedan abiertas alrededor del lugar de la ciencia política en el estudio de la violencia y la construcción del Estado-nación, así como también los retos metodológicos para abordarlas. Finalmente se hablará de la importancia del trabajo encabezado por el Grupo de Investigación en Historia de la Ciencia Política en América Latina, esbozado en el Manifiesto de Popayán, de cara al futuro de la disciplina en la región.

\section{Ciencia como objeto de indagación científica}

Por lo general puede encontrarse con alguna facilidad en colegas politólogos la misma actitud que podrían asumir los científicos más ortodoxos alrededor del tema del estudio de la ciencia como un objeto. Es decir, muchos de ellos piensan que es una pérdida de tiempo. Se asume que la ciencia no debe ser estudiada en tanto que la ciencia es la que estudia al mundo. ¿Qué de interesante puede tener el estudiar a gente que estudia? ¿Por qué no se dedican los biólogos a estudiar su objeto, es decir, la vida? ¿Por qué el politólogo no se limita a estudiar los partidos y las relaciones entre la rama ejecutiva y la legislativa? Como estas preguntas se extienden en buena parte de los colegas, no podemos hacer más que un intento por contestarlas en orden a justificar nuestra preocupación.

Lo primero que habría que decir es que el estudio de la ciencia como un objeto de indagación ya tiene cierta historia, una historia iniciada por filósofos de la ciencia y por historiadores de la ciencia. Desde finales del siglo XIX hasta la tercera década del siglo XX, la efusividad de la era moderna se manifestó en trabajos que recopilaban los hitos de la era científica, los grandes genios de la ciencia y el progreso (Nieto 1995). En buena medida esa actitud, un tanto infantil e ingenua, persiste hoy en algunos ejercicios de difusión científica, donde no se presenta mayor problematización a la idea de progreso continuo desde los "sabios y raros" griegos hasta los sofisticados científicos del presente. ${ }^{5}$ No va a ser sino hasta la década de los sesenta en que se ponga en tela de juicio esta versión del desarrollo científico, de la

5 Para una buena ilustración al respecto solo basta ver el trabajo de Carl Sagan o de su discípulo, Neil deGrasse Tyson, más conocido por su popular serie Cosmos: A Space Time Odyssey. 
mano de una emergente sociología de la ciencia que se preguntaba por las relaciones de poder y que veía a la actividad científica como una actividad humana más (Ross 1999).

El "programa fuerte" de la sociología de la ciencia, un grupo de publicaciones, especialmente británicas, de sociólogos postuló cuatro principios para abordar cualquier actividad humana alrededor del conocimiento: la causalidad, la imparcialidad, la simetría y la reflexividad. La causalidad asume que cualquier conocimiento, sea él científico o no, tiene condiciones de posibilidad donde las relaciones sociales tienen peso; la imparcialidad supone que no hay ningún criterio para diferenciar los conocimientos que pueden estudiarse, es decir, más allá de que hablemos de conocimientos verdaderos, falsos, científicos o mitológicos, no hay privilegio para ninguno. Consecuentemente con el principio anterior, la simetría sugiere que no debe haber diferencia metodológica significativa en el tratamiento de las prácticas de un laboratorio con respecto a las de una comunidad "exótica" en el corazón de la Amazonía. Finalmente, la reflexividad implica el compromiso de ejecutar esos principios para toda empresa encargada de representar al mundo, con lo que la sociología de la ciencia no es tampoco el lugar privilegiado desde el que se puede mirar sin ser observado (Nieto 1995).

Los principios mencionados han llevado a ejercicios muy interesantes sobre la práctica científica, incluso a la postulación de teorías emergentes como la TAR (Teoría del Actor Red). Un ejemplo es el trabajo de Bruno Latour, que a través de sus etnografías de laboratorios ha llegado a postular que el ejercicio científico es el producto de agencias que solo tienen sentido total cuando se descifra la red de actores humanos y no humanos que hacen que las cosas pasen. En este sentido, una verdad científica no es el descubrimiento de un hecho irrefutable, es el producto de una red de actores. Para los fines de este artículo no es necesario indagar profundamente la propuesta latouriana, pues lo que se quiere mostrar aquí es sólo una cosa: el estudio de la ciencia es mucho más de lo que podrían pensar los colegas escépticos.

Para el caso de los politólogos latinoamericanos afortunadamente ya hay un cuerpo de trabajo que puede revisarse. Pablo Bulcourf ha sintetizado esos esfuerzos junto con Nelson Cardozo y Enrique Gutiérrez en el artículo Historia y desarrollo de la ciencia política en América Latina: Reflexiones sobre la constitución del campo de estudios publicado en la "Revista de Ciencia Política" (Bulcourf, Cardozo y Gutiérrez 2015). Los autores de ese artículo hacen un recorrido por los diferentes momentos que marcan el interés de los politólogos por su propia disciplina, por la ciencia que producen. El citado interés se ha manifestado en dos fases. La primera es la fase épica, que no es otra cosa que la celebración de los grandes hitos de la ciencia y sus genios, tal como los primeros estudios sobre la historia de la ciencia. La segunda es la fase crítica, que es base del trabajo que aquí se expone, y se caracteriza por la crítica a lugares comunes de muchos politólogos a la hora de entender su disciplina y su ejercicio profesional y académico.
¿Ciencia política con sentido social? Colombia, el conflicto armado y los expertos

Juan Carlos Rico Noguera Julián Andrés Caicedo Ortiz Carlos E. Guzmán Mendoza 
Dossier América Latina: desarrollo y balance de la ciencia política
Para desarrollar un poco más lo que acaba de mencionarse, puede decirse que la fase épica vendría siendo la abierta a finales de los años noventa, una fase que se hace especialmente visible con la publicación del volumen 25 de la "Revista de Ciencia Política" en el año 2005. Bulcourf, Cardozo y Gutiérrez dirán que el trabajo realizado en esa fase se caracteriza por el desarrollo de una investigación disciplinar intensamente descriptiva que incluso da pie a la realización de trabajos comparados. Un concepto central de la fase épica de los estudios disciplinares es la institucionalización ${ }^{6}$ (Altman 2005), una certeza sería la idea de que a más democracia más ciencia política.

La fase crítica es relativamente nueva, y aunque es difícil hablar de fechas exactas, el año 2014 parece ser un buen marcador. La publicación de Caicedo, Angel y Cuellar (2014) apertura una discusión disciplinar desde los fundamentos epistémicos del conocimiento sociopolítico. El cuestionamiento directo hacia el quehacer del modelo norteamericano, excesivamente empírico-analítico, abre nuevas líneas de análisis y procura el posicionamiento de una ciencia política latinoamericana desde sus realidades y bajo sus dinámicas y aprendizajes, como lo señalado por Emmerich, Alarcón y Bulcourf, entre otros.

En el marco del tercer congreso de ACCPOL-2014 se presentó el Manifiesto de Popayán, un documento que aunque no discrimina entre los tipos de estudios disciplinares que deben hacerse, aboga especialmente por la profundización de los esfuerzos por encontrar lo que de política tiene la actividad académica del politólogo, algo que en el mejor de los casos fue inocentemente ignorado por los trabajos de la fase épica. Un trabajo especialmente brillante tanto por la forma en que se abordó como por sus duras conclusiones, es el de Paulo Ravecca. Este autor presenta evidencia de la estrecha relación existente entre los politólogos y la dictadura chilena. Incluso presenta evidencia de mayor productividad científica con respecto a la producción obtenida tras la caída de la dictadura, (Ravecca 2015). Duro golpe para las preconcepciones del politólogo promedio. También se han presentado trabajos críticos con el desarrollo de subdisciplinas específicas como la teoría política. Rico, Caicedo y Angel (2015), dos ellos coautores de este artículo, presentan evidencia para sostener que hay una abrumadora mayoría de artículos eurocéntricos de teoría política en revistas politológicas colombianas de alto impacto con respecto a artículos que desarrollen propuestas originales y ancladas a la realidad periférica del sur global. También se propone una metodología bibliográfica para hacer este tipo de seguimientos (Rico, Caicedo, Angel 2015).

En síntesis, puede decirse que más allá de la existencia de un cuerpo de trabajos dedicado a la revisión de la ciencia como un objeto de la ciencia política

6 Podría entenderse por este concepto el proceso de maduración de la ciencia política de vocación a disciplina, para ponerlo en los términos de Wills y Bejarano, antes citados. Es decir, aquel proceso de autonomización y diferenciación -en este caso, del derecho y de las otras ciencias sociales- con variadas intensidades y contenidos conforme las condiciones contextuales le favorezcan u obstaculicen. 
en América Latina, estamos entrando en un periodo interesante de revisiones críticas que pueden llevar al abandono de los lugares comunes y, quizá, a la transformación de nuestro ejercicio. La información que se presenta a continuación, extraída especialmente de la investigación realizada por Juan Carlos Rico Noguera, relaciona a la ciencia política con el conflicto armado a través del ejercicio realizado por los "expertos en violencia". Esa información permite entrever nuevas preguntas de investigación y formas de acercarse a una realidad que, como espera haberse mostrado hasta aquí, está lejos de ser una pérdida de tiempo.

\section{Estudio de la violencia en Colombia: modernización e institucionalización del Estado-nación y sus aparatos}

La comisión Gulbenkian, presidida por Immanuel Wallerstein, expuso cómo las diferentes disciplinas de la ciencia social emergen de la necesidad del Estado por conocer a su población (Wallerstein 1996). Creemos que para cualquiera debe ser evidente que el Estado no profesa precisamente intereses enciclopédicos, en esa medida la utilidad del conocimiento debía ser la de descifrar mecanismos de gobierno efectivos tanto en territorios nacionales como en territorios de ultramar. ${ }^{8}$ Por eso no debería sorprender que en el proceso de construcción del Estado-nación colombiano haya un proceso similar.

Podría decirse que el Estado-nación colombiano empieza a construirse desde la independencia formal del Reino de España. Incluso puede decirse que desde antes, cuando las élites criollas empezaron a vislumbrar sus posibilidades reales de hacer parte del gobierno colonial. Sin importar cuál sea el momento original, hay buenas razones para pensar que el Estado-nación no ha concluido su construcción, pues históricamente no ha logrado dos de los requisitos esenciales para ajustarse a ese sustantivo compuesto: en primer lugar, no ha logrado asegurar el monopolio legítimo del uso de la fuerza; en segundo lugar, no ha logrado ejercer una presencia efectiva en todo el territorio nacional (González 2003). Tal vez sea esa particularidad del Estado-nación colombiano la que hace que las comisiones de expertos alrededor de la violencia en Colombia sean tan frecuentes. En términos de ejercicios similares por unidad de tiempo en otros países, asociados por lo general a procesos de justicia transicional, no hay símil en el mundo.

La idea de comisiones de expertos empezó a calar en las élites colombianas a comienzos del siglo XX. Ya entre la década de los años veinte y la de los treinta hubo dos misiones de "asistencia técnica económica": las misiones Kemmerer (1923 y 1930). Luego, tras la inauguración del discurso global del

\footnotetext{
7 Cada vez más ligados a la ciencia política.

8 No olvidemos que el nacimiento de las ciencias sociales coincide con lo que Eric Hobsbawm denominó como la era del imperio, especialmente en Europa.
}

¿Ciencia política con sentido social? Colombia, el conflicto armado y los expertos

Juan Carlos Rico Noguera Julián Andrés Caicedo Ortiz Carlos E. Guzmán Mendoza 
Dossier América Latina: desarrollo y balance de la ciencia política desarrollo en 1948 con Estados Unidos a la cabeza, las misiones económicas en el sur global se volvieron más frecuentes (Escobar 1998), y Colombia no fue la excepción. Entre 1950 y 1958 hubo cinco misiones económicas: la Misión Currie (1950), la Misión Cepal (1954), la Misión Lebret (1955), y la segunda Misión Cepal (1958) (Arévalo 1997). Dichas misiones se entienden en el contexto de la modernización del Estado-nación colombiano, que en este caso estuvo especialmente interesado en adaptarse al capitalismo global y en hacer frente a la máxima amenaza civilizatoria de ese occidente liberal: el comunismo.

Pero en plena mitad del siglo XX Colombia no solo tenía un problema económico, tenía además un enorme problema político, pues el país se encontraba en medio de una dictadura con escasa legitimidad tanto en las élites como en buena parte de la población. Esto se debió principalmente a que el país seguía enfrascado en La Violencia9 (Palacios 2003), algo que la dictadura había buscado conjurar. También se debió a unas élites que serían capaces hasta de conciliarse con tal de volver a ostentar el control del establecimiento. El Frente Nacional, ese pacto entre élites que repartió el poder oficialmente por dieciséis años entre los partidos tradicionales (el Liberal y el Conservador) ${ }^{10}$, creó las bases de la modernización política y administrativa, una modernización que también implicó el desarrollo de las ciencias sociales en el país.

Una de las primeras políticas importantes del Frente Nacional, oficialmente lanzada el 21 de mayo de 1958, fue el nombramiento de la Comisión Nacional de las Causas y Situaciones Presentes de la Violencia en el Territorio Nacional. El objetivo de dicha comisión era establecer los motivos de la violencia interpartidista, aunque privilegiaba la concreción de medidas modernizadoras para rehabilitar las poblaciones afectadas y "atrasadas". La comisión tuvo dos momentos en el marco de su trabajo: uno de recolección de datos, en el que se aprovechó al máximo el trabajo de las comisiones económicas que la precedieron, y otro de entrevistas en campo. Lo interesante es que jamás se publicó lo hallado por la comisión en términos de las causas de la violencia interpartidista. Lo que se supo y fue expuesto por los medios de comunicación nacionales, fue el trabajo de consolidación de micropactos modernizadores regionales, algo que como Jefferson Jaramillo demuestra, se leyó como la primera política exitosa del Frente Nacional (Jaramillo 2014).

Los integrantes de la comisión fueron representantes de la élite colombiana. Ni siquiera estaban formados en disciplinas de la ciencia social, a excepción de uno solo de los comisionados. Hubo dos representantes de la Iglesia católica, dos representantes del ejército nacional, dos representantes del partido liberal, y un representante del partido conservador. ${ }^{11}$ Uno de los

\footnotetext{
9 Se escribe en mayúsculas por la connotación histórica que tiene para los colombianos.

10 Poniendo fin a casi diez años de violencia interpartidista.

${ }^{11}$ La representación del partido conservador fue menor solo porque el partido se oponía al trabajo de la comisión.
} 
representantes de la Iglesia, German Guzmán Campos, fue el cientista social que gracias a su trabajo en la comisión y su mancomunada publicación de $L a$ violencia en Colombia ${ }^{12}$ fortaleció el desarrollo de la ciencia social nacional, publicación que por mucho ha sido, y será, la referencia de trabajo sociológico por excelencia (Jaramillo 2014).

La ciencia política no existía para el momento en que la comisión del Frente Nacional realizó su trabajo, de hecho aparecería en la Universidad de los Andes, universidad privada, diez años después de su lanzamiento. Probablemente el hecho de aparecer en una universidad privada con una agenda especialmente ceñida al curso disciplinar de los Estados Unidos (Bejarano, Wills 2005), de un lado, y de contar para su desarrollo con un equipo de profesores que no tenían compromisos políticos al estar vinculados al gobierno, del otro (Duque Daza 2014), alejaría a la disciplina del estudio de la violencia por un buen tiempo, hasta finales del siglo XX.

La segunda comisión de expertos de la violencia tuvo lugar en 1987. Tras treinta años de políticas modernizantes los problemas eran diferentes. La relativa pacificación del país, o más bien, la transformación de la violencia, llevó a nuevos asuntos. La Comisión de Estudios sobre la Violencia se encargó de estudiar los nuevos formatos de la violencia, asociados a la lucha insurgente y a la aparición de la violencia callejera y los déficits democráticos. En este caso la mayor parte de los comisionados eran cientistas sociales que tenían como un punto de referencia el trabajo de Guzmán, Fals Borda y Umaña. Tenían también en común el hecho de pertenecer a universidades públicas de varias partes del país, pero especialmente a la Universidad Nacional de Colombia en Bogotá.

La Comisión de Estudios sobre de la Violencia fue el primer encargo de gran magnitud que el Estado le hizo a personas formadas para la investigación social, algo que generó duras críticas por parte de académicos que afirmaban la necesidad de una ciencia social independiente del Estado (Villaveces 1998). Dentro de los comisionados no había ningún politólogo de formación. Sin embargo, sí hubo algunos comisionados con posgrados en ciencia política, como fue el caso de Eduardo Pizarro y Carlos Miguel Ortiz.

Toda la investigación, esta vez sin un trabajo de campo directo, se realizó en las instalaciones del IEPRI (Instituto de Estudios Políticos y Relaciones Internacionales) de la Universidad Nacional de Colombia en Bogotá. Aunque alimentada por algunas investigaciones de campo, quedó la sensación de un trabajo poco sensible a las particularidades regionales de las nuevas violencias, a pesar de que haya habido una serie de aportes originales en el marco de los estudios de la violencia para el momento (Jaramillo 2014). La voz de los directamente implicados en los fenómenos violentos no apareció.

No obstante, cabe señalar que el hecho de que la investigación hubiese sido desarrollada en el IEPRI fue significativo para una ciencia política emergente
¿Ciencia política con sentido social? Colombia, el conflicto armado y los expertos

Juan Carlos Rico Noguera Julián Andrés Caicedo Ortiz Carlos E. Guzmán Mendoza

\footnotetext{
12 Junto con Orlando Flas Borda y Eduardo Umaña Luna.
} 
Dossier América Latina: desarrollo y balance de la ciencia política dentro de la Universidad Nacional. Después de todo, se reconoce el trabajo del IEPRI como el origen de la disciplina en esa universidad (Bejarano, Wills 2005). Eduardo Pizarro fue uno de sus miembros fundadores del IEPRI. Otro elemento importante por mencionar de cara a la relación entre esta comisión y la emergencia de la ciencia política en el país es su preocupación por el tema de la democracia. Buena parte del informe de la comisión se dedica a reflexionar alrededor de las carencias de la democracia colombiana, presentando así recomendaciones para su profundización. De acuerdo con algunos de los comisionados, sus recomendaciones fueron recogidas en algunos aspectos por el trabajo de la asamblea constituyente que dio lugar a la constitución de 1991 (Jaramillo 2014).

La siguiente comisión que vale la pena mencionar es la Comisión Nacional de Reparación y la Reconciliación (CNRR) nacida en el año 2005. Hubo varias comisiones antes de la CNRR, pero ninguna introdujo tantas novedades como esta. En términos del proceso de modernización del Estado, puede decirse que se articula a lo que se ha conocido en los debates de justicia transicional a nivel internacional como "el viraje a la verdad" (Teitel 2003). El viraje a la verdad es la consecuencia de las dificultades para afrontar pasados violentos para distintos sistemas de justicia en el marco de las transiciones a la democracia del final del siglo XX. La memoria y la reparación simbólica adquieren una importancia protagónica en ese viraje. Adicionalmente, la justicia transicional se impone sobre la ordinaria en orden a asegurar el éxito de las transiciones a la democracia, sobre todo cuando el pasado es de dictadura (Teitel 2003). En el caso de los países en conflicto, como el colombiano, "el viraje a la verdad" funciona como un mecanismo que desactiva el conflicto, repara a las víctimas con acceso a la verdad, la justicia y la reparación, asegurando al mismo tiempo la consolidación del proyecto de Estado-nación (Hayner 2011).

La CNRR funcionaba con varios delegados de diferentes estamentos del Estado. El representante de la vicepresidencia de la república fue uno de los actores centrales del desarrollo de la ciencia política en el país: Eduardo Pizarro. Pizarro creó el Grupo de Memoria Histórica (GMH) junto con Gonzalo Sánchez, quien hubiera sido el director de la comisión de 1987. El GMH, que en su momento fue una subdivisión de la CNRR, es ahora una organización estatal autónoma que funcionará unos años más.

El trabajo de los miembros del GMH ha revolucionado los estudios de la violencia en el país, pues se pasó de la narrativa experta a lo que Alejandro Castillejo llamaría "los archivos del dolor" (Castillejo 2013). Estos "archivos del dolor" no son otra cosa que la recopilación sistemática de testimonios sobre la victimización. Esta recopilación hace del trabajo algo mucho menos violento $^{13}$, pues no hay imposiciones de sentido desde el centro a la periferia. La investigación es, en buena medida, guiada por los testimonios de las víc-

13 Siguiendo la noción de violencia epistémica propuesta por Gayarti Chakravorty Spivak (Spivak 2003). 
timas, aunque haya problemas (Alfonso, Beristain 2013). Esto para la ciencia política tradicional no es precisamente común o aceptado, sin embargo, no son pocos los miembros del GMH relacionados con la disciplina.

Para empezar, el director general, Gonzalo Sánchez, ha sido uno de los investigadores más notables del IEPRI que, como se mencionó ya, es entendido como uno de los organismos fundadores de la ciencia política en el país. Álvaro Villarraga Sarmiento, director de la dirección de recursos para la verdad es politólogo. Martha Nubia Bello, directora del Museo Nacional de la Memoria (todavía un proyecto) tiene posgrado en ciencia política. Asesores externos del CNMH como Maria Emma Wills y Andrés Suarez también están relacionados con la disciplina, sin contar con los muchos empleados que no hacen parte de los perfiles principales del grupo, un dato que valdría la pena revisar.

Una última comisión que debe abordarse es la más reciente: la Comisión Histórica del Conflicto y sus Víctimas (CHCV). Esta comisión, producto de las negociaciones de paz entre el gobierno de Colombia y el más importante grupo guerrillero del país, las FARC, se encargó de tres tareas: definir los orígenes y múltiples causas del conflicto armado interno, definir los múltiples factores que han perpetuado el conflicto, y definir los efectos e impactos más notorios del conflicto sobre la población. Esta comisión fue una suerte de retorno a la comisión del 87 , pues se construyó sobre la exclusiva narrativa experta, pero hay diferencias notables.

En primer lugar, la CHCV no presentó un informe conjunto, tal vez de ahí el apelativo que los mismos comisionados le daban: la comisión pluralista (Pizarro, Gómez 2014). Tan pluralista fue que hubo 12 ensayos independientes y dos relatorías. La comisión del 87 creó un consenso entre todos los comisionados y presentó un informe unificado. En segundo lugar, la $\mathrm{CHCV}$ fue requerida tanto por el gobierno como por las FARC, haciendo incluso que la escogencia de comisionados se dividiera entre las partes negociadoras (Mesa de Conversaciones 2014). En este punto habría cosas interesantes por definir en la medida de que es la primera vez que ocurre en el país y que ha habido mucha cautela frente al tema. En tercer lugar, hay un claro aumento de "población politológica”, aunque representa a solo dos ciudades del país: Bogotá y Medellín. Maria Emma Wills, Gustavo Duncan, Jairo Estrada, Francisco Gutierrez, Vicente Torrijos y Eduardo Pizarro, la mitad de los expertos, tienen fuertes vínculos con la ciencia política.

Exceptuando a Eduardo Pizarro, que participó como uno de los relatores, todos los académicos que se nombraron arriba hicieron su propia lectura de los tres elementos que debían aclararse en el marco de la comisión. Pese a que esos académicos están vinculados a la ciencia política, no hubo precisamente lecturas muy cercanas a lo que sería el mainstream de la disciplina, algo apenas entendible, teniendo en cuenta que el estudio del conflicto ha sido algo mucho más propio de otras disciplinas, como la sociología, la historia y hasta la antropología. Tal vez lo más cercano a ese mainstream sería lo realizado por Duncan y por Torrijos, ambos cercanos a los discursos de la seguridad
¿Ciencia política con sentido social? Colombia, el conflicto armado y los expertos

Juan Carlos Rico Noguera Julián Andrés Caicedo Ortiz Carlos E. Guzmán Mendoza 
Dossier
América Latina:
desarrollo y balance
de la ciencia política especialmente desarrollados en los estudios estratégicos de las relaciones internacionales.

Tras esta revisión de las comisiones de estudio sobre la violencia, puede verse que en efecto el camino de modernización estatal ha sido acompañado por cientistas sociales que a través de su trabajo en las comisiones de estudio de la violencia han intentado incidir en la profundización de la democracia liberal. También puede verse que los politólogos han ido incrementando su injerencia en este proceso modernizador del Estado, algo que puede estar relacionado con el rápido crecimiento de la disciplina. Sin embargo, como acaba de sugerirse, sería difícil sostener que la implicación de la ciencia política signifique la realización de trabajos mainstream. Por el contrario, parece haber aquí la semilla de una ciencia política muy cercana a la interdisciplinaridad y al trabajo académico con sentido social. Este sentido social sería el de consolidar la construcción del Estado-nación liberal, algo que desde otros esfuerzos también liderados por politólogos se traduce en "la construcción de la paz".

Pero ya se ha dicho al principio de este artículo que lo que resuena con esta relación de la ciencia política con el conflicto armado y la construcción del Estado-nación no es precisamente algo enteramente positivo, puede leerse incluso como un canto de sirena. La razón de lo anterior es que se ha ignorado por completo a la región periférica. Es difícil entender cómo es que los académicos que hacen parte de las comisiones que se han repasado en este apartado hagan parte de contextos tan lejanos del conflicto armado en Colombia. A pesar de que el departamento del Cauca tiene su propia universidad con académicos en diferentes áreas de la ciencia social, incluida la ciencia política, todavía no ha habido comisionados de esa región del país, por ejemplo. Esto es particularmente problemático, ya que esa es una de las regiones más golpeadas por el conflicto. El trabajo realizado por académicos vinculados a la región podría arrojar resultados aclaradores.

\section{Consideraciones metodológicas de los fenómenos nombrados y los que se aproximan}

Hay muchos elementos de los fenómenos que se han venido presentando que deben ser estudiados. Lo que hasta ahora se ha presentado es el producto de una investigación de archivo, y en muchas ocasiones esta no basta para responder preguntas fundamentales de cualquier estudio de la ciencia. Por ejemplo ¿Por qué se eligen expertos de unas regiones en vez de expertos de otras? Aunque nos podríamos quedar con la sospecha, bien fundada en términos históricos, de una estructura de poder colonial, un estudioso de la ciencia no puede quedarse sencillamente con la sospecha. Confirmar esa estructura de poder, en el caso de la CHCV, implicaría un trabajo de entrevistas a actores clave, que van desde los comisionados hasta miembros de los equipos negociadores del gobierno y las FARC. 
Sin embargo, existen más preguntas importantes. ¿Cómo se constituyen las narrativas de los expertos? ¿Por qué las narrativas y prácticas expertas en el caso de las últimas comisiones colombianas se parecen a las desarrolladas por la comisión sudafricana de la verdad de 1995? ¿Cómo se trazan los vínculos entre ciertos expertos y un Estado-nación en construcción? Todas estas preguntas ameritan más que entrevistas y trabajos de archivo. En este sentido, el trabajo etnográfico parece una respuesta factible. El trabajo etnográfico en el marco de los estudios de la ciencia ha venido entregando respuestas muy sugerentes alrededor de las prácticas expertas. Bruno Latour tiene sus famosas etnografías de laboratorios (Latour, Woolgar 1995) mientras la investigadora Karen Ho tiene su influyente trabajo alrededor de las prácticas de Wallstreet (Ho 2009). Ambos ejemplos, que son pocos para una práctica investigativa cada vez más extendida en distintos ámbitos de la ciencia y el saber experto, pueden dar luces para la investigación de corte disciplinar que se ha venido haciendo desde el Grupo de Investigación en Historia de la Ciencia Política en América Latina.

\section{El Manifiesto de Popayán y el futuro de la ciencia política latinoamericana}

Llegados a este punto vale la pena recordar la importancia del Manifiesto de Popayán para la ciencia política en América Latina. El Manifiesto de Popayán es el compromiso alrededor de la profundización de la investigación disciplinar de varios investigadores que se realizó durante el tercer congreso de la ACCPOL. Tal compromiso tiene mucho de útil para el desarrollo de la disciplina, pues no solo implica revisar y celebrar los diferentes momentos de la ciencia política en la región, como tal vez fue el caso de la fase épica de los estudios disciplinares. Implica sobre todo la comprensión del lugar de los politólogos en el mundo, implica incluso la comprensión de un fenómeno tan central para la ciencia política como la formación del Estado-nación.

Ya se había dicho que una de las grandes ganancias de la fase crítica de los estudios disciplinares es salir de los lugares comunes que plagan a la politología. ¿Qué tal si empezamos a valorar, más allá de los partidos y las instituciones formales, el lugar de sujetos tan influyentes como los expertos en la construcción de los proyectos políticos de la modernidad? ¿Qué tal si nos damos cuenta que el politólogo es mucho más que un investigador y lo vemos como uno de los nodos de una red más grande que impulsa el cambio histórico?

Creemos que el Manifiesto de Popayán tiene la posibilidad de transformar el trabajo de la ciencia política latinoamericana en la medida de que nos permite plantear las preguntas que acabamos de plantear. Lo creemos también porque más allá de plantearlas, nos permite pensar en cómo responderlas. 


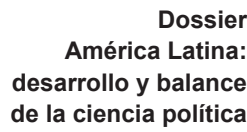

de la ciencia política

\section{Conclusiones}

Quisiéramos formular algunas preocupaciones a modo de provocación. Provocación que tiene como propósito incentivar el debate frente a la necesidad de investigar la ciencia política desde la ciencia, utilizando las herramientas, instrumentos y perspectivas epistemológicas, propias de las ciencias sociales.

En este sentido, en primer lugar, bien podría afirmarse que luego de casi medio siglo de haberse iniciado la ciencia política en el país, nuestro sistema político ha observado importantes transformaciones sociales, económicas $y$, por supuesto, políticas. Transformaciones que han ido de la mano, en buena medida, del proceso de institucionalización disciplinar. El Estado-nación colombiano es uno distinto a aquel de 1968, cuando se creó el primer programa de ciencia política. Aunque políticamente más sólido, no por ello es exento de riesgos y amenazas derivadas, principal, aunque no exclusivamente, de la tradición política que hereda el pasado violento y clientelista del sistema.

Coligado con lo anterior, la disciplina en la actualidad se encuentra firmemente arraigada en el contexto universitario nacional. Más desconcentrada y diversificada en su oferta, tanto a nivel de pregrado como de postgrado. Hay más investigación, principalmente en los subcampos disciplinares, pero con importantes debates, discusiones y aportes desde la ciencia para la ciencia política. Hay, del mismo modo, más revistas y publicaciones derivadas de los esfuerzos investigativos que, individual o colectivamente, se hacen tanto por parte de los centros de pensamiento tradicionales y centrales, como desde los regionales. También más espacios para la discusión y debate de las aportaciones que desde uno y otro lugar se publican. Hoy, siguiendo a Duque Daza (2014), la disciplina politológica en Colombia cuenta con los elementos que caracterizan "la institucionalización disciplinar", pero no por ello consideramos que hemos alcanzado una plena "institucionalización".

Reiteramos la riqueza y utilidad del trabajo etnográfico en el marco de los estudios de la ciencia. Principalmente, las respuestas, muy sugerentes alrededor de las prácticas expertas, que este nos ofrece.

Finalmente, vale señalar que el Manifiesto de Popayán es una invitación. Extendida a todos aquellos expertos y no expertos politólogos que crean que la disciplina no agota su objeto de estudio en los subcampos delimitados formalmente como campos de acción e investigación. Es, al mismo tiempo, compromiso con el loable propósito de continuar -desde el centro, desde la periferia, pero principalmente desde la misma ciencia- en al arduo camino de la investigación disciplinar de la ciencia política colombiana. Creemos, firmemente que el Manifiesto de Popayán tiene la posibilidad de aportar en la transformación del trabajo politológico colombiano y, porque no de América Latina. La posibilidad de plantearnos preguntas que se convierten en retos, como las que acabamos de plantear, y muchas otras que se hagan en la misma perspectiva; y, pensar en cómo responderlas es, por ahora, nuestro mejor argumento. 


\section{Bibliografía}

Alfonso C., Beristain C. (2013), Memoria para la vida. Una comisión de la verdad desde las mujeres para Colombia, Ruta Pacífica de las Mujeres, Bogotá.

Altman D. (2005), La institucionalización de la ciencia política en Chile y América Latina: una mirada desde el sur, "Revista de Ciencia Política", vol. 25(1), pp. 3-15.

Arévalo D. (1997), Misiones Económicas Internacionales en Colombia 1930-1960, "Historia Crítica", vol. 14, pp. 7-24.

Bejarano A. M., Wills M. E. (2005), La ciencia política en Colombia: de vocación a disciplina, "Revista de Ciencia Política", vol. 25(1).

Bulcourf P., Cardozo N., Gutiérrez E. (2015), Historia y desarrollo de la ciencia política en América Latina: Reflexiones sobre la constitución del campo de estudios, "Revista de Ciencia Política”, vol. 35(1), pp. 179-199.

Castillejo A. (2013), Los archivos del dolor. Ensayos sobre la violencia y el recuerdo en la Sudáfrica contemporánea, Ediciones Uniandes, Bogotá.

Duque Daza J. (2014), La ciencia política en Colombia. La construcción de una comunidad académica, Universidad del Valle, Cali.

Escobar A. (1998), La invención del Tercer Mundo. Construcción y deconstrucción del desarrollo, Norma, Bogotá.

Garcé A. (2015), El institucionalismo discursivo como oportunidad: La ciencia política latinoamericana y el estado del arte en la literatura sobre el poder político de las ideas, "Política y Gobierno", vol. 22(1), pp. 199-226.

González F. (2003), ¿Colapso parcial o presencia diferenciada del Estado en Colombia?: una mirada desde la historia, "Colombia Internacional", vol. 58, pp. 124-159.

Hayner P. (2011), Unspeakable Truths: Transitional Justice and the Challenge of Truth Commissions, Routledge, New York.

Ho K. (2009), Liquidated: An Ethnography of Wall Street, Duke University Press.

Jaramillo J. (2014), Pasados y presentes de la violencia en Colombia. Estudios sobre las comisiones de investigación (1958-2011), Editorial Pontificia Universidad Javeriana, Bogotá.

Latour B., Woolgar S. (1995), La vida en el laboratorio. La construcción de los hechos científicos, Alianza Editorial, Madrid.

Leyva Botero S., Fortou J. A., Preciado A. F., Ramírez M. F. (2013), Ciencia política en Colombia: una revisión de la literatura sobre el estado e historia de la disciplina en el país, en: La ciencia política en Colombia ¿una disciplina en consolidación?, S. Leyva Botero (ed.), Colciencias, Asociación Colombiana de Ciencia Política, Centro de Análisis Político - Universidad Eafit, Medellín.Mesa de Conversaciones (2014, agosto 5), Comunicado Conjunto, La Habana, 05 de agosto de 2014, disponible en: https://www.mesadeconversaciones.com.co/comunicados/ comunicado-conjunto-la-habana-05-de-agosto-de-2014?ver=es.

Nieto M. (1995), Poder y conocimiento científico: nuevas tendencias en historiografía de la ciencia, "Historia Crítica", enero-junio, pp. 3-14.

Palacios M. (2003), Entre la legitimidad y la violencia. Colombia 1958-2010, Norma, Bogotá.

Pizarro E., Gómez M. (2014, febrero 14), Gobierno y FARC coinciden: «la lucha armada ha sido inútil», "El tiempo", Bogotá, disponible en: http://www.eltiempo.com/politica/
¿Ciencia política con sentido social? Colombia, el conflicto armado y los expertos

Juan Carlos Rico Noguera Julián Andrés Caicedo Ortiz Carlos E. Guzmán Mendoza 
Dossier América Latina: desarrollo y balance de la ciencia política proceso-de-paz/informe-de-la-comision-historica-del-conflicto-habla-uno-de-losrelatores/15249518.

Ravecca P. (2015), Our discipline and its politics. Authoritarian political science: Chile 19791989, "Revista de Ciencia Política", vol. 35(1), pp. 145-178.

Rico J. C., Caicedo J. A., Angel S. (2015), Colonialidad del saber y ciencias sociales: una metodología para aprehender los imaginarios colonizados, "Análisis Político", vol. 85, pp. 76-92.

Ross A. (1999), The Challenge of Science, en: The Cultural Studies Reader, Routledge, London, pp. 292-307.

Spivak G. C. (2003), ¿Puede hablar el subalterno?, "Revista Colombiana de Antropología”, vol. 39, pp. 297-364.

Teitel R. (2003), Transitional Justice Genealogy, "Harvard Human Rights Journal”, vol. 16, pp. 69-94.

Varsavsky O. (2010), Ciencia, política y cientificismo y otros textos, Capital Intelectual, Buenos Aires.

Villaveces S. (1998), Entre pliegues de ruinas y esperanzas. Viñetas sobre estudios de la violencia en el IEPRI, "Análisis Político", vol. 34, pp. 80-98.

Wallerstein I. (1996), Abrir las ciencias sociales: informe de la Comisión Gulbenkian para la reestructuración de las ciencias sociales, Siglo XXI. 